\title{
Compressed and split spectra in minimal SUSY SO(10)
}

\section{Frank F. Deppisch ${ }^{1 *}$, Nishita Desai ${ }^{2}$ and Tomás E. Gonzalo ${ }^{1}$}

${ }^{1}$ Department of Physics and Astronomy, University College London, London, UK

${ }^{2}$ Institut für Theoretische Physik, Universität Heidelberg, Heidelberg, Germany

\section{Edited by:}

Stefano Morisi, University of

Wurzburg, Germany

Reviewed by:

Ilja Doršner, University of Sarajevo,

Bosnia and Herzegovina

Sudhir Kumar Vempati, Indian

Institute of Science, India

\section{*Correspondence:}

Frank F. Deppisch, Department of

Physics and Astronomy, University

College London, D106B, Physics

Building, Gower Street, London

WC1E 6BT, UK

e-mail:f.deppisch@ucl.ac.uk

\section{INTRODUCTION}

The non-observation of new heavy states at the LHC puts strong constraints on the sparticle spectrum of supersymmetric (SUSY) theories, especially in the colored sector. Most importantly, this puts a strain on the ability of many SUSY models to solve the hierarchy problem of the Standard Model (SM) in a natural fashion. In minimal scenarios, such as the constrained minimal supersymmetric Standard Model (CMSSM), the stringent lower limits on colored states will similarly affect non-colored sparticles. The direct LHC search limits on these sparticle species as well as third generation squarks are on the other hand comparatively weak and can depend strongly on the details of the spectrum. Various solutions have been suggested to resolve the constraints and generate viable and testable scenarios. For example, phenomenological approaches like the phenomenological MSSM (pMSSM) do not contain a priori relations between different sparticle species and can be constructed to avoid the strong constraints but still provide states that can be produced at the LHC in the near future. On the other hand, such approaches often lack motivation.

In this work, we focus on a minimal supersymmetric $\mathrm{SO}(10)$ model [1-3] incorporating one-step symmetry breaking from $\mathrm{SO}(10)$ down to the Standard Model gauge group at the usual Grand Unified Theory (GUT) scale $M_{\mathrm{GUT}} \approx 2 \cdot 10^{16} \mathrm{GeV}$ where the SM gauge couplings unify within an MSSM spectrum. Such a framework is therefore well motivated: It not only incorporates gauge unification but the unification of matter fields in a 16-plet would also provide degenerate soft SUSY breaking scalar masses at the GUT scale. In this scenario, the soft SUSY breaking sector is given by the gravity induced mass parameters for the matter and Higgs superfields at the GUT scale. Being a subset of the MSSM at low energies, two Higgs fields are required to generate masses separately for up- and down-type fermions during electroweak symmetry breaking. In the $\mathrm{SO}(10)$ framework, these Higgs fields are generally produced from the superposition of doublet components in a set of Higgs fields at the GUT scale $[4,5]$. In the present analysis, we do not discuss the issue of
Yukawa unification. Successful Yukawa unification of all fermion generations in $\mathrm{SO}(10)$ either requires a set of Higgs fields in large representations [4-7] or the presence of Planck-scale suppressed higher-dimensional operators $[8,9]$.

In contrast to the CMSSM with its strictly degenerate soft scalar mass spectrum at the GUT scale, the scalar masses in the minimal SUSY SO(10) are non-universally shifted by D-terms associated with the breaking of $\mathrm{SO}(10)$ to the lower-rank SM group [10-12]. These D-terms are analogous to the electroweak D-terms in the MSSM due to the rank reducing breaking of the SM gauge group. As described below in section 2, the $\mathrm{SO}(10)$ D-terms depend on the details of the breaking of $\mathrm{SO}(10)$ but are generally expected to be of the order of the SUSY breaking scale. They can therefore have a sizable impact on the sparticle spectrum. The possible presence of the $\mathrm{SO}(10)$ D-terms represents the main deviation from the CMSSM case, and we will analyze their impact on the sparticle spectrum in light of the LHC searches. As opposed to the phenomenological models, the non-degeneracy is not ad hoc and can be described by the introduction of a single additional parameter $m_{D}^{2}$. Starting at the GUT scale, the non-degenerate scalar masses evolve, following the renormalization group (RG) of the MSSM [13] down to the electroweak scale. This results in a sparticle spectrum at the supersymmetry scale chosen at $1 \mathrm{TeV}$ according to the SPA convention [14]. If these masses were to be observed at the LHC or at other future colliders, the reverse RG evolution upwards would allow the reconstruction of the physics scenario at the GUT scale [15-21].

In addition to the non-universality of scalar masses at the GUT scale due to $\mathrm{SO}(10) \mathrm{D}$-terms, we also allow for a non-degeneracy of the fermionic masses of the gauginos. While the gauge couplings unify at the GUT scale, the gauginos only do so if the messenger mediating the breaking of SUSY in a hidden sector is an $\mathrm{SO}(10)$ singlet [22]. This is not required though, and the messenger can be part of various $\mathrm{SO}(10)$ representations, provided it remains a singlet under the SM gauge groups. 
This paper is organized as follows: In section 2 we introduce the minimal SO(10) framework and the main consequences on the sparticle spectrum due to possible large D-terms and non-unification of the gaugino masses. Section 3 reviews the relevant direct sparticle mass limits from recent LHC searches. The results of our renormalization group analysis are presented in section 4 and we summarize our conclusions in section 5 .

\section{SUSY SO(10)}

SUSY GUT models are largely fixed by their gauge group structure. In $\mathrm{SO}(10)$, a generation of the SM fermions is contained in a 16 representation with the addition of a right-handed neutrino. Variations are then induced by the choice of the breaking of the GUT group to the $\mathrm{SM}$ group $\mathrm{SU}(3)_{c} \times \mathrm{SU}(2)_{L} \times \mathrm{U}(1)_{Y}$. There are numerous ways in which this symmetry breaking can occur. A minimum of two breaking steps are required: one to break $\mathrm{SO}(10)$ to the SM group at a high scale $M_{\mathrm{GUT}} \approx 2 \times 10^{16} \mathrm{GeV}$ (where the SM gauge couplings unify in the MSSM), and one to break the electroweak symmetry of the SM at $M_{\mathrm{EW}}$. Among all the different possible breaking paths from $S O(10)$ to $S U(3) \otimes S U(2) \otimes U(1)$, displayed in Figure 1, we will adopt the minimal path labeled (a). It should be noted that for phenomenological purposes, this is equivalent to multi-step breaking scenarios close to the scale $M_{G U T}$.

The electroweak Higgs fields of the MSSM are contained in higher-dimensional representations of $\mathrm{SO}(10)$, which couple to the SM fermions via Yukawa-type interactions. The only allowed representations for this field, given the $\mathrm{SO}(10)$ group structure, are $\mathbf{1 0}, \mathbf{1 2 0}$, and $\mathbf{1 2 6}$. We do not consider non-renormalizable operators which broaden the range of allowed Higgs representations. The simplest choice is to use the $\mathbf{1 0}$ dimensional representation containing the electroweak Higgs fields. These choices motivate the superpotential

$$
W_{\mathrm{SO}(10)}=\mathbf{Y}_{16} 1 \mathbf{1 0}_{H} 16_{F}+\mu_{H} 10_{H} 10_{H}+W(\Sigma),
$$

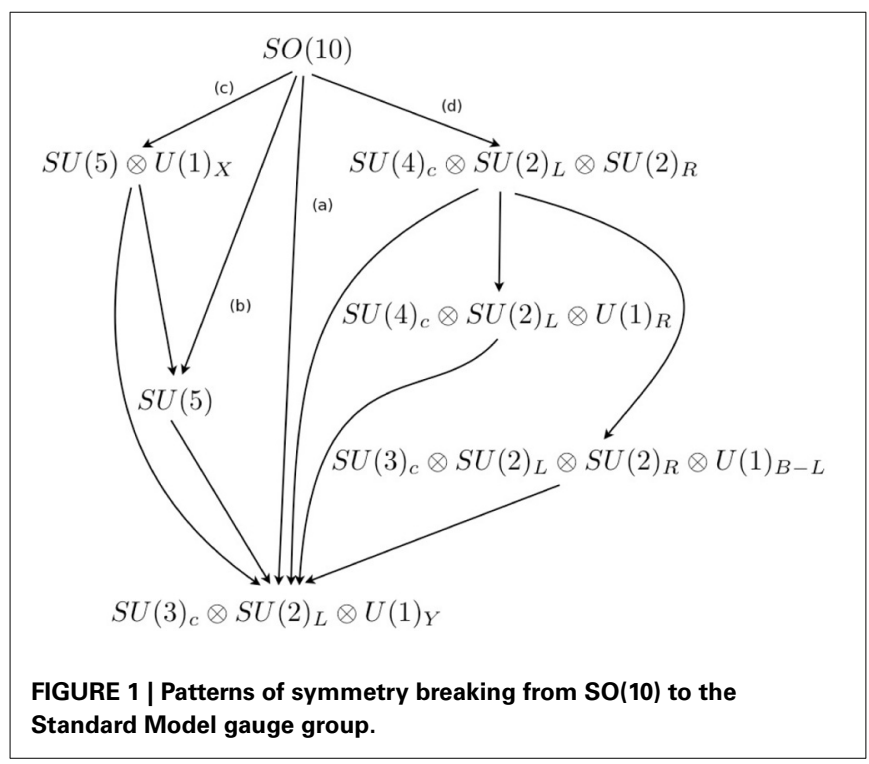

where $\mathbf{Y}$ is a $3 \times 3$ matrix in generation space. The term $W(\Sigma)$ collects all terms that involve the Higgs field(s) $\Sigma$ responsible for $\mathrm{SO}(10)$ breaking, which we can neglect in our low energy analysis.

The Higgs sector described above, i.e., the $\mathrm{SO}(10)$ breaking Higgs and the $\mathbf{1 0}_{H}$ containing the EW breaking Higgses, is not enough to predict the masses of all fermions in a Yukawa unified scenario. One would need to add larger representations and/or higher-dimensional operators, as mentioned before. However, extending this sector would not have a significant effect for the purpose of this study, for it is mostly focused on sfermion masses and any contribution coming from an extended Higgs sector can be neglected to the level of approximation at which we are working.

As phenomenologically required, SUSY has to be broken and the generated soft-SUSY breaking sector will depend on the particular breaking mediation mechanism. We assume Supergravity (SUGRA) mediated SUSY breaking where SUSY is broken above the GUT scale in a hidden particle sector. Before $\mathrm{SO}(10)$ breaking, these terms take the form

$$
\begin{aligned}
\mathcal{L}_{\text {soft }}= & -\mathbf{m}_{16_{F}}^{2} \tilde{16}_{F}^{*} \tilde{16}_{F}-m_{10_{H}}^{2} 10_{H}^{*} 10_{H} \\
& -\frac{1}{2} m_{1 / 2} \tilde{X} \tilde{X}-A_{0} \mathbf{Y} \tilde{1} \sigma_{F} \tilde{16}_{F} 10_{H}-B_{0} \mu_{H} 10_{H} 10_{H}+\text { c.c. } \\
& +\mathcal{L}_{\Sigma},
\end{aligned}
$$

where $\tilde{X}$ represents the gaugino field, $\tilde{16} \sigma_{F}$ and $10_{H}$ refer to the scalar components of the $\mathbf{1 6}_{F}$ and $\mathbf{1 0}_{H}$ superfields, respectively. The corresponding soft breaking masses are denoted as $m_{1 / 2}$, $\mathbf{m}_{16_{F}}^{2}$ (in general a $3 \times 3$ matrix in generation space) and $m_{10_{H}}^{2}$, respectively. The term c.c. stands for complex conjugate and $\mathcal{L}_{\Sigma}$ collects any operators containing the $\Sigma$ field, which are irrelevant for our discussion. The SUSY breaking equivalents of the Yukawa coupling and Higgs $\mu$-term are controlled by the common trilinear coupling $A_{0}$ and $B_{0}$, respectively. In the following we will adopt the standard CMSSM boundary conditions for the trilinear soft-SUSY breaking parameters in the MSSM at the GUT scale:

$$
A_{u}=A_{d}=A_{e}=A_{0} .
$$

The corresponding boundary conditions for the soft scalar and gaugino masses will be discussed below.

\subsection{SCALAR D-TERMS}

The scalar potential of the $\mathrm{SO}(10)$ model, responsible for the symmetry breaking, is obtained from the scalar parts of the superpotential in Equation (1) plus the scalar soft breaking terms of Equation (2). In addition, there is an extra contribution that arises from the so called D-terms of the Kähler potential [11]. Such Dterms are generated during gauge symmetry breaking that reduces the rank of the original group, i.e., when one or more of the embedded $U(1)$ subgroups is broken. The most prominent example is the electroweak D-term generated in the MSSM through the electroweak symmetry breaking (EWSB) of the SM gauge group to $\mathrm{SU}(3) \times \mathrm{U}(1)_{Q}$. For the breaking of a single $\mathrm{U}(1)$ subgroup, the process can be described as follows: The field acquiring a vacuum expectation value, $\Sigma$ in our case, has components with 
opposite charges under this U(1) subgroup, $\Phi$ and $\Phi\left(H_{u}\right.$ and $H_{d}$ for EWSB). After symmetry breaking and after integrating out the heavy $\Phi$ and $\Phi$, scalar particle masses receive contributions of the form Kolda and Martin[11]

$$
\Delta m_{i}^{2}=Q_{i} m_{D}^{2}, \quad \text { with } \quad m_{D}^{2}=\frac{1}{2} \frac{\left(\bar{m}^{2}-m^{2}\right)}{Q_{\Phi}}
$$

where $Q_{i}$ and $Q_{\Phi}$ are the charges of the light scalar particle species $i$ and the $\Phi$ field under the broken $U(1)$, respectively. The soft masses of the $\Phi$ and $\bar{\Phi}$ fields are given by $m$ and $\bar{m}$, respectively, and they are related to the soft mass of the $\Sigma$ field(s) in Equation (2). The D-term $m_{D}^{2}$ will therefore be roughly of the same order as the soft masses instead of the GUT scale where the breaking actually occurs. For more complicated breaking scenarios, the dependence of $m_{D}^{2}$ on the soft masses will vary slightly, according to the Higgs representation(s) involved, but it will still remain of the same order. In the case of EWSB, a linear combination of the $\mathrm{U}(1)_{Y}$ and the $\mathrm{U}(1)$ included in $\mathrm{SU}(2)_{L}$, generated by the $I_{3}$ generator, is broken. The electroweak D-terms has the value [23]

$$
\Delta m_{i}^{2}=M_{Z}^{2} \cos 2 \beta\left(I_{3}^{i}-Q_{i} \sin \theta_{W}\right),
$$

with the third component of the weak isospin $I_{3}^{i}$ and the charge $Q_{i}$ of sparticle $i[\tan \beta$ is the usual ratio of Higgs vacuum expectation values (VEVs)].

The contributions from the $\mathrm{SO}(10)$ D-term changes the boundary conditions for the scalar masses at the GUT scale. When the symmetry is spontaneously broken, the MSSM scalar masses match the $\mathrm{SO}(10)$ soft breaking masses in Equation (2), plus the contributions from the D-term. Assuming that all soft-SUSY masses are diagonal and universal in generation space, the boundary conditions for the MSSM soft masses $\mathbf{m}_{Q}^{2}, \mathbf{m}_{u}^{2}, \mathbf{m}_{e}^{2}, \mathbf{m}_{L}^{2}, m_{H_{d}}^{2}, m_{H_{u}}^{2}$ read [4-11]

$$
\begin{aligned}
\mathbf{m}_{Q}^{2} & =\mathbf{m}_{u}^{2}=\mathbf{m}_{e}^{2}=m_{16_{F}}^{2} \mathbf{1}+m_{D}^{2} \mathbf{1}, \\
\mathbf{m}_{L}^{2} & =\mathbf{m}_{d}^{2}=m_{16_{F}}^{2} \mathbf{1}-3 m_{D}^{2} \mathbf{1}, \\
\mathbf{m}_{v}^{2} & =m_{16_{F}}^{2} \mathbf{1}+5 m_{D}^{2} \mathbf{1} \\
m_{H_{d}}^{2} & =m_{10_{H}}^{2}+2 m_{D}^{2} \\
m_{H_{u}}^{2} & =m_{10_{H}}^{2}-2 m_{D}^{2} .
\end{aligned}
$$

The coefficients in front of $m_{D}^{2}$ correspond to the $\mathrm{U}(1)$ charges of the different sparticles. This Abelian $\mathrm{U}(1)$ group is embedded into $\mathrm{SO}(10)$ via $\mathrm{SU}(5) \otimes \mathrm{U}(1) \subset \mathrm{SO}(10)$ and thus all particles in the same representation of $\mathrm{SU}(5)$ will have the same charge. For completeness, we have also stated the boundary condition for the right-handed sneutrino soft mass $\mathbf{m}_{v}^{2}$. In the following, we will not consider the right-handed sneutrino as part of our spectrum. We implicitly assume it acquires a mass close to the GUT scale in a neutrino seesaw framework, and neglect the effect it could have on the running of the other sparticles as well as the lepton flavor violation it induces in the slepton sector. These effects depend delicately on the details of the neutrino sector. Equation (6) describes the crucial impact of the presence of an $\mathrm{SO}(10) \mathrm{D}$-term. Most importantly it will cause a splitting between the sparticle species $\tilde{Q}, \tilde{u}, \tilde{e}$ and $\tilde{L}, \tilde{d}$ already at the GUT scale. This D-term induced splitting will be increased through RGE running, potentially causing a split spectrum at the low scales.

The D-term will in general depend on the vacuum expectation value of the field that breaks the $\mathrm{SO}(10)$ gauge group, which in turn is related to the soft SUSY breaking masses as can be seen in the example Equation (4). The specific value of the term depends very strongly on the scalar potential of the $\mathrm{SO}(10)$ breaking sector, but because we want to keep our description as independent as possible from the GUT scale physics, we will parameterize this by allowing $m_{D}$ to be a free parameter in our model. Thus, provided that the Yukawa couplings are fixed by the fermion masses up to the ratio of electroweak VEVs $\tan \beta=v_{u} / v_{d}$, and the $B_{0}$ and $\mu_{H}$ parameters are obtained by imposing electroweak vacuum stability conditions, the only free parameters of our model relevant to low energy phenomenology are

$$
m_{16_{F}}^{2}, m_{10_{H}}^{2}, m_{D}^{2}, m_{1 / 2}, A_{0}, \tan \beta, \operatorname{sign}\left(\mu_{H}\right)
$$

Figure 2 shows how the masses of the first generation sfermions are split due the effect of the D-term. In order to present the dependence on the D-term $m_{D}^{2}$ in a convenient way, we define the function

$$
\sigma\left(m_{D}^{2}\right)=\operatorname{sign}\left(m_{D}^{2}\right) \sqrt{\left|m_{D}^{2}\right|}
$$

The rest of the model parameters are fixed by using the benchmark scenario provided in Table 1 of Buchmueller et al. [24],

$$
\begin{aligned}
m_{16_{F}} & =1380 \mathrm{GeV}, \quad m_{10_{H}}=3647 i \mathrm{GeV}, \quad m_{1 / 2}=3420 \mathrm{GeV}, \\
A_{0} & =-3140 \mathrm{GeV}, \quad \tan \beta=39, \quad \operatorname{sign}\left(\mu_{H}\right)=1,
\end{aligned}
$$

corresponding to a non-universal Higgs mass (NUHM1) high scale scenario.

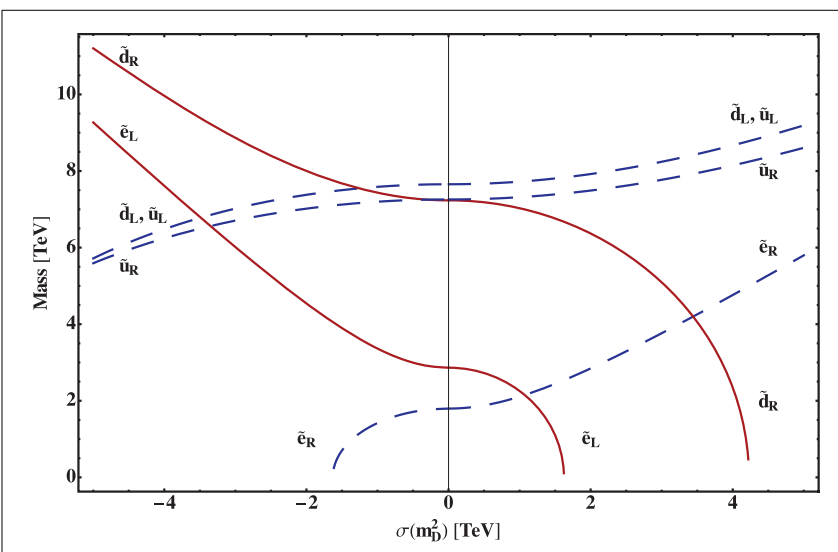

FIGURE 2 | First generation sfermion masses as a function of the SO(10) D-term $\sigma\left(m_{D}^{2}\right)=\operatorname{sign}\left(m_{D}^{2}\right) \sqrt{\left|m_{D}^{2}\right|}$. The values for the other model parameters are fixed as in Equation. (9). 


\subsection{NON-UNIVERSAL GAUGINO MASSES}

A standard assumption of the CMSSM is the unification of the gaugino masses at the GUT scale to the common value $m_{1 / 2}$ in Equation (2). This is not necessarily true for more general SUSY breaking mechanisms. In particular, the $\mathrm{SO}(10)$ representation of the SUSY-breaking mediator field determines the matching conditions at the GUT scale. The field is required to be a singlet under the SM in order to preserve its symmetry but it does not need to be a singlet under $\mathrm{SO}(10)$. Table 1 shows different boundary conditions for a selection of possible representations of the mediating field [22]. In the simplest case, the mediator field is in the singlet representation, in which case the matching conditions at the GUT scale are:

$$
M_{1}=M_{2}=M_{3}=m_{1 / 2} .
$$

Other choices can have advantages, such as improved Yukawa unification [25]. Other examples are models with negative $\mu_{H}$ which can be made compatible with the experimental value of the anomalous magnetic moment of the muon, $a_{\mu}$, by making $\mu M_{2}$ positive through the choice of a configuration with negative $M_{2}$ from Table 1.

In models that undergo gauge mediated supersymmetry breaking, this non-universality emerges naturally at the messenger scale due to the nature of the breaking. At this messenger scale, usually around or above $10^{6} \mathrm{GeV}$, the masses of gauginos are induced by one-loop corrections involving messenger fields, and are of the form Dine and Nelson [26]

$$
M_{a}=\frac{\alpha_{a}}{4 \pi} \Lambda \sum_{n_{a}} n_{a},
$$

where $\Lambda$ is the relative splitting of the fermionic and scalar parts of the messenger superfields (source of supersymmetry breaking) and $n_{a}$ is the Dynkin index of the messenger fields in the SM subgroup $a$. In this case there can be two sources of non-universality: first, there is a natural splitting due to the different values of the gauge couplings $\alpha_{a}$, and second, the sum of the Dynkin indices could naively be different for the three gauge groups. However, if

Table 1 | Ratios of gaugino masses for a SUSY breaking messenger field in different representations of $S U(5) \subset S O(10)$ [22] at the GUT

\begin{tabular}{|c|c|c|c|c|c|}
\hline \multirow[t]{2}{*}{ So(10) } & \multirow[t]{2}{*}{$S U(5)$} & \multicolumn{2}{|c|}{$M_{\mathrm{GUT}}$} & \multicolumn{2}{|c|}{$M_{\mathrm{EW}}$} \\
\hline & & $\frac{M_{1}}{M_{3}}$ & $\frac{M_{2}}{M_{3}}$ & $\frac{\overline{M_{1}}}{M_{3}}$ & $\frac{M_{2}}{M_{3}}$ \\
\hline $1,54,210,770$ & 1 & 1 & 1 & $\frac{1}{6}$ & $\frac{1}{3}$ \\
\hline $54,210,770$ & 24 & $\frac{1}{2}$ & $-\frac{3}{2}$ & $-\frac{1}{12}$ & $-\frac{1}{2}$ \\
\hline 210,770 & 75 & -5 & 3 & $-\frac{5}{6}$ & 1 \\
\hline 770 & 200 & 10 & 2 & $\frac{5}{3}$ & $\frac{2}{3}$ \\
\hline
\end{tabular}
and the EW scale.

The EW ratios take into account the approximate effect of the RGE running on the gaugino masses. these messengers come in complete representations of the unified group (in order to preserve the unification of gauge couplings), the sum of the Dynkin indices is the same for all three gauginos. In this case, the only splitting at the messenger scale comes from the different values of $\alpha_{a}$, which can be rather small, and depends mostly on the messenger scale. In this paper we will focus only on mSUGRA-inspired scenarios, where the only non-universality in the gaugino mass comes from the $\mathrm{SO}(10)$ representation of the mediator field. Unless otherwise stated, we will consider universal gauginos at the GUT scale, with mSUGRA induced supersymmetry breaking. The effect of having non-universal gauginos on the particle spectrum will be studied in section 4.3.

\subsection{RENORMALIZATION GROUP EVOLUTION}

Below the GUT scale, with the heavy gauge bosons and Higgs fields integrated out, the particle content of the minimal SUSY $\mathrm{SO}(10)$ model is the same as in the MSSM. We implicitly assume that the right-handed neutrinos and sneutrinos also decouple at or close to the GUT scale within a seesaw framework of light neutrino mass generation. Therefore the Renormalization Group Equations (RGEs) will be same as those of the MSSM but with different boundary conditions at the GUT scale. The complete RGEs for the MSSM and their approximate solutions are listed in Appendix A in Supplementary Material. In this section we will focus on the relevant consequences for the sparticle spectrum in the minimal SUSY $\mathrm{SO}(10)$ model using appropriate approximations.

The RGEs for the scalar masses of the first two generations can be exactly solved at one loop by neglecting small Yukawa couplings. For the very same reason, there is no mixing between the left and right-handed squarks or sleptons under such an approximation. The RGEs are then given by

$$
\begin{aligned}
& 16 \pi^{2} \frac{d}{d t} m_{Q_{1,2}}^{2}=-\frac{32}{3} g_{3}^{2} M_{3}^{2}-6 g_{2}^{2} M_{2}^{2}-\frac{2}{15} g_{1}^{2} M_{1}^{2}+\frac{1}{5} g_{1}^{2} S, \\
& 16 \pi^{2} \frac{d}{d t} m_{u_{1,2}}^{2}=-\frac{32}{3} g_{3}^{2} M_{3}^{2}-\frac{32}{15} g_{1}^{2} M_{1}^{2}-\frac{4}{5} g_{1}^{2} S, \\
& 16 \pi^{2} \frac{d}{d t} m_{d_{1,2}}^{2}=-\frac{32}{3} g_{3}^{2} M_{3}^{2}-\frac{8}{15} g_{1}^{2} M_{1}^{2}+\frac{2}{3} g_{1}^{2} S, \\
& 16 \pi^{2} \frac{d}{d t} m_{L_{1,2}}^{2}=-6 g_{2}^{2} M_{2}^{2}-\frac{6}{5} g_{1}^{2} M_{1}^{2}-\frac{3}{5} g_{1}^{2} S, \\
& 16 \pi^{2} \frac{d}{d t} m_{e_{1,2}}^{2}=-\frac{24}{5} g_{1}^{2} M_{1}^{2}+\frac{6}{5} g_{1} S,
\end{aligned}
$$

with the gauge couplings $g_{i}$ and gaugino masses $M_{i}$. The term $S$ is defined as

$$
S=m_{H_{u}}^{2}-m_{H_{d}}^{2}+\operatorname{Tr}\left(m_{Q}^{2}-2 m_{u}^{2}+m_{d}^{2}-m_{L}^{2}+m_{e}^{2}\right)
$$

Although $S$ has a dependence on all the scalar masses, this particular combination turns out to be exactly solvable, and the solution depends only on the gauge couplings and the value of $S$ at the GUT scale. However, in the case that all scalar masses are universal, i.e., have the same value at the GUT scale, this term vanishes. It therefore has the role of quantifying the non-universality of a model. In our particular case, the universality is violated due to the appearance of the D-term, and so the only contribution left 
from this $S$ term is proportional to $m_{D}^{2}$. Thus the masses for all first and second generation squarks and sleptons can be expressed analytically as [20]

$$
\begin{aligned}
& m_{\tilde{u}_{L}}^{2}=m_{16_{F}}^{2}+m_{D}^{2}\left(1+2 C_{1}^{(1)}\right)+m_{1 / 2}^{2}\left(C_{3}^{(2)}+C_{2}^{(2)}+\frac{1}{6} C_{1}^{(2)}\right)+D_{u_{L}}, \\
& m_{\tilde{u}_{R}}^{2}=m_{16_{F}}^{2}+m_{D}^{2}\left(1-8 C_{1}^{(1)}\right)+m_{1 / 2}^{2}\left(C_{3}^{(2)}+\frac{8}{3} C_{1}^{(2)}\right)+D_{u_{R}}, \\
& m_{\tilde{d}_{L}}^{2}=m_{16_{F}}^{2}+m_{D}^{2}\left(1+2 C_{1}^{(1)}\right)+m_{1 / 2}^{2}\left(C_{3}^{(2)}+C_{2}^{(2)}+\frac{1}{6} C_{1}^{(2)}\right)+D_{d_{L}}, \\
& m_{\tilde{d}_{R}}^{2}=m_{16_{F}}^{2}+m_{D}^{2}\left(-3+4 C_{1}^{(1)}\right)+m_{1 / 2}^{2}\left(C_{3}^{(2)}+\frac{2}{3} C_{1}^{(2)}\right)+D_{d_{R}}, \\
& m_{\tilde{e}_{L}}^{2}=m_{16_{F}}^{2}+m_{D}^{2}\left(-3-6 C_{1}^{(1)}\right)+m_{1 / 2}^{2}\left(C_{2}^{(2)}+\frac{3}{2} C_{1}^{(2)}\right)+D_{e_{L}}, \\
& m_{\tilde{e}_{R}}^{2}=m_{16_{F}}^{2}+m_{D}^{2}\left(1+12 C_{1}^{(1)}\right)+m_{1 / 2}^{2}\left(6 C_{1}^{(2)}\right)+D_{e_{R}}, \\
& m_{\tilde{v}_{L}}^{2}=m_{16_{F}}^{2}+m_{D}^{2}\left(-3-6 C_{1}^{(1)}\right)+m_{1 / 2}^{2}\left(C_{2}^{(2)}+\frac{3}{2} C_{1}^{(2)}\right)+D_{v_{L}}, \quad(14)
\end{aligned}
$$

where the $C_{a}^{(n)}$ are constants, defined as

$$
\begin{aligned}
C_{a}^{(n)} & =\frac{c_{a}}{b_{a}}\left(1-\frac{g_{a}^{2 n}\left(M_{\mathrm{SUSY}}\right)}{g_{a}^{2 n}\left(M_{\mathrm{GUT}}\right)}\right), \quad\left(c_{1}, c_{2}, c_{3}\right)=\left(\frac{1}{5}, \frac{3}{2}, \frac{8}{3}\right), \\
\left(b_{1}, b_{2}, b_{3}\right) & =\left(\frac{33}{5}, 1,-3\right),
\end{aligned}
$$

The electroweak D-terms $D_{i}$ are defined in Equation (5) and they are usually sub-dominant to the soft scalar masses.

The constants $C_{a}^{(n)}$ depend only on the gauge couplings. However, there is a non-trivial dependence on $\tan \beta$ within the electroweak D-terms. Since they are essentially negligible, we fix $\tan \beta$ to the value in the benchmark scenario described in Equation (9), $\tan \beta=39$. The scalar masses for the 1 st and 2nd generation squarks and sleptons can then be numerically written as

$$
\begin{aligned}
& m_{\tilde{u}_{L}}^{2}=m_{16_{F}}^{2}+1.0 m_{D}^{2}+5.3 m_{1 / 2}^{2}-(53.6 \mathrm{GeV})^{2}, \\
& m_{\tilde{u}_{R}}^{2}=m_{16_{F}}^{2}+0.9 m_{D}^{2}+4.9 m_{1 / 2}^{2}-(35.8 \mathrm{GeV})^{2}, \\
& m_{\tilde{d}_{L}}^{2}=m_{16_{F}}^{2}+1.0 m_{D}^{2}+5.3 m_{1 / 2}^{2}+(59.3 \mathrm{GeV})^{2}, \\
& m_{\tilde{d}_{R}}^{2}=m_{16_{F}}^{2}-2.9 m_{D}^{2}+4.9 m_{1 / 2}^{2}+(25.3 \mathrm{GeV})^{2}, \\
& m_{\tilde{e}_{L}}^{2}=m_{16_{F}}^{2}-3.1 m_{D}^{2}+0.5 m_{1 / 2}^{2}+(47.3 \mathrm{GeV})^{2}, \\
& m_{\tilde{e}_{R}}^{2}=m_{16_{F}}^{2}+1.2 m_{D}^{2}+0.2 m_{1 / 2}^{2}+(43.9 \mathrm{GeV})^{2}, \\
& m_{\tilde{v}_{L}}^{2}=m_{16_{F}}^{2}-3.1 m_{D}^{2}+0.5 m_{1 / 2}^{2}-(64.5 \mathrm{GeV})^{2} .
\end{aligned}
$$

For illustration, Figure 3 shows the running of the scalar masses in a representative example scenario. As the usual MSSM RGE running is driven by the gaugino mass $m_{1 / 2}$, the additional impact of the $\mathrm{SO}(10) \mathrm{D}$-term is roughly determined by the ratio $m_{D}^{2} / m_{1 / 2}^{2}$. For $m_{D}^{2} / m_{1 / 2}^{2} \ll 1$, the spectrum will be of the usual CMSSM type, whereas for $m_{D}^{2} / m_{1 / 2}^{2} \gtrsim 1$, the impact of the $\mathrm{SO}(10) \mathrm{D}$-term on the sparticle spectrum will be sizeable.

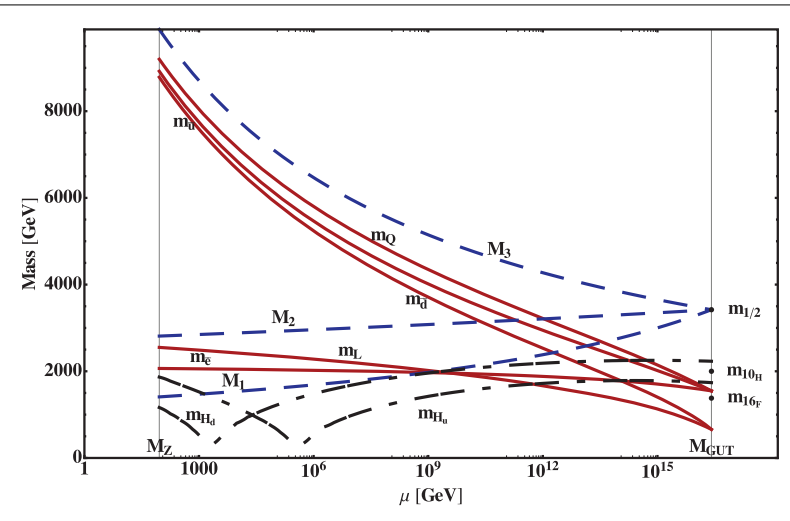

FIGURE 3 | Solution of the RGEs for the scalar masses of the 1st generation, the gaugino masses and the Higgs doublet masses in the benchmark scenario defined in Equation (9) but with $m_{D}^{2}=(0.7 \mathrm{TeV})^{2}$ and $m_{10_{H}}^{2}=(2 \mathrm{TeV})^{2}$.

Different sparticle masses in the Equations $(14,16)$ depend on the model parameters $m_{16_{F}}^{2}, m_{D}^{2}$, and $m_{1 / 2}$ with the same or very similar coefficients. We use this to construct linear combinations of these masses that depend on a reduced number of parameters, which will become very useful when trying to find an optimal scenario in the parameter space. The first combination to consider is among the particles belonging to different multiplets in the SU(5) subgroup of $\mathrm{SO}(10)$. Due to the presence of the $\mathrm{D}$-terms this combination will induce a large splitting between the left and right handed squarks and sleptons, given by

$$
\begin{aligned}
& m_{\tilde{d}_{L}}^{2}-m_{\tilde{d}_{R}}^{2}=3.9 m_{D}^{2}+0.4 m_{1 / 2}^{2}+\mathcal{O}\left(M_{Z}^{2}\right) \\
& m_{\tilde{e}_{L}}^{2}-m_{\tilde{e}_{R}}^{2}=-4.3 m_{D}^{2}+0.3 m_{1 / 2}^{2}+\mathcal{O}\left(M_{Z}^{2}\right) .
\end{aligned}
$$

Secondly, the splitting between those masses with similar D-term contributions, i.e., those supersymmetric particles that belong to the same multiplet in the $\mathrm{SU}(5)$ subgroup of $\mathrm{SO}(10)$ is given by

$$
\begin{aligned}
& m_{\tilde{d}_{R}}^{2}-m_{\tilde{e}_{L}}^{2}=0.2 m_{D}^{2}+4.4 m_{1 / 2}^{2}+\mathcal{O}\left(M_{Z}^{2}\right), \\
& m_{\tilde{u}_{L}}^{2}-m_{\tilde{e}_{R}}^{2}=-0.2 m_{D}^{2}+5.1 m_{1 / 2}^{2}+\mathcal{O}\left(M_{Z}^{2}\right), \\
& m_{\tilde{u}_{R}}^{2}-m_{\tilde{e}_{R}}^{2}=-0.3 m_{D}^{2}+4.7 m_{1 / 2}^{2}+\mathcal{O}\left(M_{Z}^{2}\right)
\end{aligned}
$$

These splittings are largely driven by the gauge contributions proportional to $m_{1 / 2}$ also present in the CMSSM. Nevertheless, a large $\mathrm{SO}(10) \mathrm{D}$-term $m_{D}^{2}$ can appreciably contribute to the splitting for small $m_{1 / 2}$.

Thirdly, a small splitting is caused by the EW D-terms in the left-handed squarks and the left-handed sleptons, which, belonging to the same $S U(2)$ multiplet, are quasi-degenerate, with a splitting proportional to $M_{Z}^{2}$,

$$
\begin{aligned}
& m_{\tilde{d}_{L}}^{2}-m_{\tilde{u}_{L}}^{2}=\mathcal{O}\left(M_{Z}^{2}\right), \\
& m_{\tilde{e}_{L}}^{2}-m_{\tilde{v}_{L}}^{2}=\mathcal{O}\left(M_{Z}^{2}\right) .
\end{aligned}
$$


The above relations are obtained by using only the 1-loop solution of the RGEs which may not be accurate for large values of $m_{D}^{2}$. We calculate the 2-loop corrections using the approximation discussed in Appendix A in Supplementary Material and find that these contributions are, at most,

$$
\begin{aligned}
\left(\delta m_{2 \text {-loop }}^{2}\right)_{1,2}< & \mathcal{O}\left(10^{-2}\right)\left(-m_{16_{F}}^{2}-m_{1 / 2}^{2}\right) \\
& +\mathcal{O}\left(10^{-3}\right)\left(-m_{10_{H}}^{2}-m_{D}^{2}\right) \\
\left(\delta m_{2 \text {-loop }}^{2}\right)_{3}< & \mathcal{O}\left(10^{-2}\right)\left(-m_{16_{F}}^{2}-m_{1 / 2}^{2}\right) \\
& +\mathcal{O}\left(10^{-3}\right)\left(-m_{10_{H}}^{2}-m_{D}^{2}+A_{0}^{2}+A_{0} m_{1 / 2}\right),
\end{aligned}
$$

for the first two and the third generations, respectively. As expected, for large values of the parameters these contributions can be significant and hence we will take them into account in our analysis.

\section{DIRECT SUSY SEARCHES AT THE LHC 3.1. REINTERPRETATION OF SOUARK AND GLUINO LIMITS}

The most stringent limits on superpartner masses currently come from searches for strongly charged superpartners viz. squarks and gluons. LHC searches based on multiple jets and missing energy currently rule out squarks masses of the order of $2 \mathrm{TeV}$ and gluino masses of the order of $1 \mathrm{TeV}$ depending on the model used for interpretation $[27,28]$. In this section, we determine how these limits translate to the SUSY SO(10) parameters.

The supersymmetric $\mathrm{SO}(10)$ model has two parameters that affect the squark masses at tree level, $m_{16}$ and $m_{D}^{2}$. In particular, a non-zero $m_{D}^{2}$ results in a split between left- and right-handed squarks. Therefore, the simplification in the CMSSM that all squarks of the first two generations are nearly degenerate is lost. For this analysis, we have retained the universal gaugino sector, meaning the gaugino masses originate from a common parameter at the GUT scale leading to a ratio $M_{1}: M_{2}: M_{3}=1: 2: 6$ at the electroweak scale.

We factorize the problem of estimating final cross section after the cuts into two steps. Firstly, we analytically calculate the production cross section and the branching fractions. Secondly, we estimate the efficiencies of the cuts in each production mode for the jets+MET search channels reported by ATLAS using Monte Carlo simulation.

The efficiency of the cuts is calculated using a simplified model with two parameters $m_{\tilde{g}}$ and $m_{\tilde{q}}$. There are four production modes that result in jets+MET final states viz. $\tilde{g} \tilde{g}, \tilde{q} \tilde{q}, \tilde{q} \tilde{q}^{*}$ and $\tilde{q} \tilde{g}$. We assume each squark decays as $\tilde{q} \rightarrow q \tilde{\chi}_{1}^{0}$ and the gluino decays via either $\tilde{g} \rightarrow q \tilde{q}$ if $m_{\tilde{g}}>m_{\tilde{q}}$ or via $\tilde{g} \rightarrow q \bar{q} \tilde{\chi}_{1}^{0}$ otherwise. As a consistency check, we reproduce the ATLAS limits based on [27] for a simplified model where all squarks are degenerate and the lightest (bino-dominated) neutralino is the LSP with a mass a sixth of the gluino mass. The comparison is shown in Figure 4, where the CMSSM model with all squarks being degenerate $\left(\tilde{u}_{L}\right.$, $\left.\tilde{d}_{L}, \tilde{u}_{R}, \tilde{d}_{R}\right)$ is plotted in green and the observed ATLAS limit in dashed black. The Monte-Carlo simulation was performed using Pythia 8 [29-31] with Gaussian smearing of the momenta of the jets and leptons as a theorist's detector simulation. Figure 4

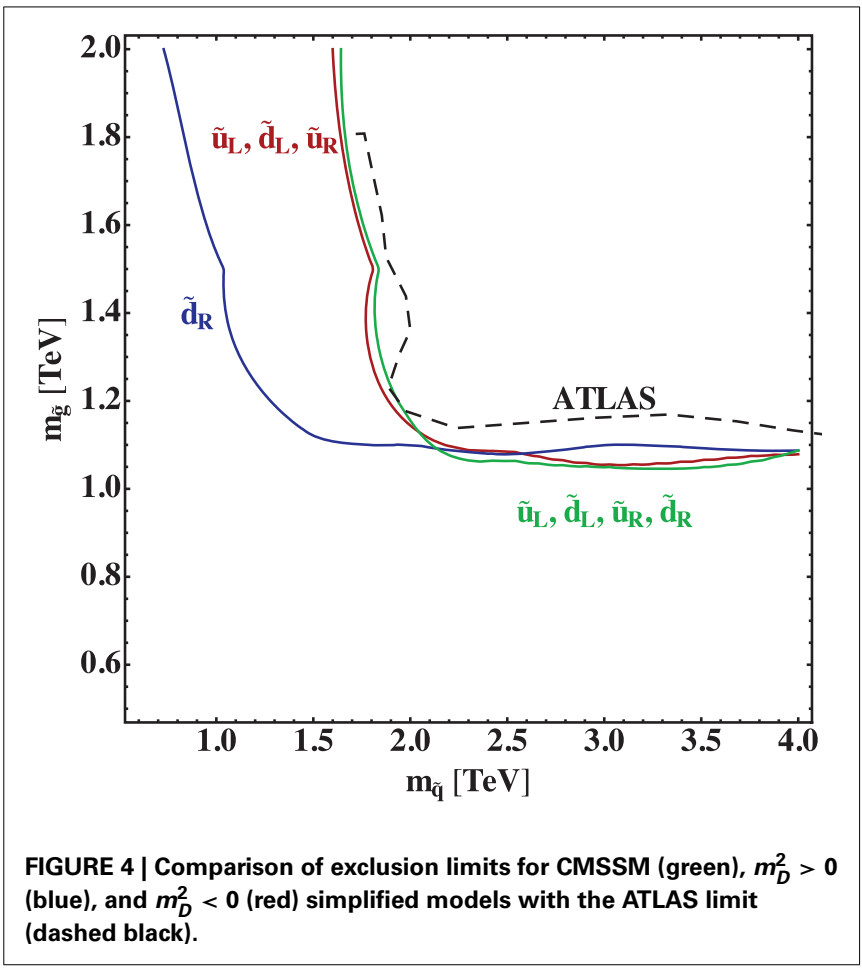

demonstrates that we approximately reproduce the exclusion limit reported by ATLAS in our simulation.

To investigate the change in the ATLAS limits given a non-zero $m_{D}^{2}$, we use two separate simplified models. First, corresponding to $m_{D}^{2} \gg 0$, we have the case where right-handed, down-type squarks are much lighter than the rest. We approximate this by setting $m_{\tilde{d}_{R}}=m_{\tilde{s}_{R}}=m_{\tilde{b}_{1}}=m_{\tilde{q}}$ and all other squark masses set to $10 \mathrm{TeV}$. Second, corresponding to $m_{D}^{2} \ll 0$, we have the case where all left-handed squarks along with the right-handed uptype quarks are light. This is approximated by a simplified model where $m_{\tilde{q}}$ corresponds to the degenerate mass of all squarks except the ones in the $m_{D}^{2} \gg 0$ model. The change in the exclusion limit for both of these cases is also shown in Figure 4, where the $m_{D}^{2} \gg 0\left(\tilde{d}_{R}\right.$ light $)$ case is plotted in blue, and $m_{D}^{2} \ll 0$ case $\left(\tilde{u}_{L}\right.$, $\tilde{d}_{L}$ and $\tilde{u}_{R}$ light $)$ is in red. The exclusion limit in the case $m_{D}^{2} \ll 0$ is almost identical to the fully degenerate CMSSM case, whereas $m_{D}^{2} \gg 0$ leads to a considerably weaker limit $m_{\tilde{q}} \gtrsim 1 \mathrm{TeV}$. The gluino limit remains unaffected.

Assuming a similar sensitivity with $300 \mathrm{fb}^{-1}$ integrated luminosity at the $14 \mathrm{TeV}$ run of the LHC, we expect to rule out up to $m_{\tilde{q}} \sim 3.2 \mathrm{TeV}$ for the $m_{D}^{2} \ll 0$ case and $m_{\tilde{q}} \sim 2.8 \mathrm{TeV}$ for the $m_{D}^{2} \gg 0$ case. The reach in gluino mass is about $m_{\tilde{g}} \sim 3.6 \mathrm{TeV}$. A 3 -sigma discovery can be made for $m_{\tilde{q}} \sim 2.5 \mathrm{TeV}$ for the $m_{D}^{2} \ll 0$ case and $m_{\tilde{q}} \sim 1.8 \mathrm{TeV}$ for the $m_{D}^{2} \gg 0$ case. We have added a comment in this regard in section 2.3.

\subsection{SUMMARY OF OTHER LHC SUSY SEARCHES}

After the first run of the LHC, a great amount of the data has been analyzed and comprehensive searches for supersymmetric signals have been carried out. Both ATLAS and CMS have done 
an extensive survey of many different scenarios and studied the data collected in the most model independent way possible, so as to exclude as much of the SUSY parameter space as possible. We summarize here the exclusion limits for some of the supersymmetric particles:

\subsubsection{Stops and sbottoms}

Stops are produced at the LHC mostly through the s-channel, and the primary decay modes are $\tilde{t} \rightarrow t \tilde{\chi}^{0}$ and $\tilde{t} \rightarrow b \tilde{\chi}^{ \pm}$. The final states studied have the signature $4 j+l+M E T$, with none to three $b$-tags and the current lower limit on the stop mass is around $m_{\tilde{t}} \gtrsim 650 \mathrm{GeV}$. However, if the stop is not allowed to decay to an on-shell top, $m_{\tilde{t}}<m_{t}+m_{\tilde{\chi}^{0}}$, the decay phase space is reduced and the process is suppressed which weakens the limit to $m_{\tilde{t}} \gtrsim 250 \mathrm{GeV}$. Searches for sbottoms are similar to those for stops, with similar production rates and complementary decays, $\tilde{b} \rightarrow b \tilde{\chi}^{0}$ and $\tilde{b} \rightarrow t \tilde{\chi}^{ \pm}$. Consequently, the mass limits are similar, $m_{\tilde{b}} \gtrsim 650 \mathrm{GeV}$ [32-36].

\subsubsection{Sleptons, neutralinos, and charginos}

Although electroweak processes at the LHC are several orders of magnitude smaller than strong ones, the precision of the measurements done by ATLAS and CMS is good enough to provide a limit of $m_{\tilde{l}} \gtrsim 300 \mathrm{GeV}$. Similar to the sleptons, the limits on the neutralinos and charginos are considerably weaker than those of gluinos and squarks. Using purely electroweak processes such as $\tilde{\chi}_{2}^{0} \tilde{\chi}^{ \pm} \rightarrow Z \tilde{\chi}^{0} W^{ \pm} \tilde{\chi}^{0}$ or $\tilde{\chi}_{2}^{0} \tilde{\chi}^{ \pm} \rightarrow l \tilde{v} \tilde{l} l(v \tilde{v})$, both LHC experiments have currently excluded masses up to $m_{\tilde{\chi}} \gtrsim 300 \mathrm{GeV}$ [37-40]. Finally, the extra Higgs states predicted by supersymmetry have also been subject to scrutiny. However, due to the strong dependence on the parameters in the MSSM (particularly $\tan \beta$ ), the limits are not very strong. As of today, the limits seem to favor $\tan \beta \gtrsim 18$ and Higgs masses around or above that of the found Higgs state, $m_{H, A, H^{ \pm}} \gtrsim 100 \mathrm{GeV}$ [41-44].

\section{ANALYSIS}

The SUSY SO(10) model has seven free parameters, $m_{16_{F}}^{2}$, $m_{10_{H}}^{2}, m_{1 / 2}, m_{D}^{2}, A_{0}, \tan \beta, \operatorname{sign}(\mu)$, when no constraints are imposed. We will use existing experimental limits to fix or constrain some of these model parameters using the results of section 3 , focusing on the most interesting deviations from the standard CMSSM scenario.

As discussed above, there is a lower limit on the mass of the lightest squark, at $m_{\tilde{q}} \gtrsim 2 \mathrm{TeV}$ within the framework of the CMSSM. With the degeneracy of all scalar particles at the GUT scale, this bound also forces the sleptons to become heavy, usually well beyond the direct detection slepton mass limits. However, in the minimal SUSY $\mathrm{SO}(10)$ model, it is possible to evade the squark limits while keeping the slepton masses light, possibly at the level of experimental detectability. We will therefore seek to explore the model parameter space with a large splitting between the squark and slepton masses by taking advantage of the relation (18). Even in the CMSSM, one may obtain relatively light sleptons (compared to squarks) by increasing the RG running effect of the strong gauge coupling by increasing $m_{1 / 2}$. A large value of $m_{1 / 2}$ is actually required due to the corresponding gluino mass limit $m_{\tilde{g}} \gtrsim 1 \mathrm{TeV}$. For a fixed squark mass, this approach has the disadvantage that it will also raise the lightest neutralino mass which is the preferred Lightest Supersymmetric Particle (LSP) candidate. In order to have the lightest neutralino lighter than any charged sparticle for as much of the parameter space as possible, we will fix the value of $m_{1 / 2}$ so as to produce a gluino with a mass roughly at the current limit, $m_{\tilde{g}} \approx 1 \mathrm{TeV}$.

The only other free parameter in Equation (18) is $m_{D}^{2}$, which has a comparatively small contribution toward the splitting. This is because the scalar species under consideration belong to the same SU(5) multiplets and the splitting is caused by a secondary effect in the RGEs. Notice also that the splitting for the $\overline{5}$ and 10 multiplets has opposite signs in their dependence on $m_{D}^{2}$, cf. Equation (17), i.e., for $m_{D}^{2} \gg 0, \tilde{e}_{L}, \tilde{d}_{R}$ will be the lighter states whereas for $m_{D}^{2} \ll 0$ it will be $\tilde{e}_{R}$ and $\tilde{u}_{L}$.

We will therefore look for a region of parameter space where, by increasing $m_{D}^{2}$ in both positive and negative directions, we achieve a large splitting between squarks and sleptons. Since $m_{1 / 2}$ is fixed, as stated above, and in order to keep the mass of the lightest first generation squark $\left(m_{\tilde{q}}\right)$ fixed to the lowest allowed value, we express $m_{16_{F}}^{2}$ as a function of the other model parameters and the desired squark mass $m_{\tilde{q}}$,

$$
m_{16_{F}}^{2}=m_{\tilde{q}}^{2}-c_{1} m_{D}^{2}-c_{2} m_{1 / 2}^{2}-c_{3}+\delta_{2}
$$

where the constants $c_{i}$ are taken from Equation (16) for the corresponding squark species and $\delta_{2}$ is the 2-loop correction to the mass of the lightest squark. The latter is significant for large $\left|m_{D}^{2}\right|$ and $m_{16_{F}}^{2}$. The limit of this procedure is reached as soon as one of the particles becomes tachyonic (negative squared mass) at the electroweak scale.

Due to the large third generation Yukawa couplings, especially for the top quark, the third generations of sparticles are usually lighter than the first two. We will consider this case first in the following section. In section 4.2 , we will describe the possibility of having the first two generations lighter than the third by compensating the RG effect of the Yukawa couplings. To conclude, in section 4.3 , we will study the additional impact of non-universal gauginos on the sparticle spectrum.

\subsection{LIGHT THIRD GENERATION}

Starting with the benchmark scenario described in Equation (9), and parameters set by the current LHC limits we will perform a scan over $m_{D}^{2}$ to analyze how the masses of different sparticles behave. To achieve a light but viable SUSY spectrum, the value of $m_{1 / 2}$ is fixed such that $m_{\tilde{g}}=1 \mathrm{TeV}$ at the current exclusion limit. The value of $m_{16_{F}}^{2}$ is then determined so as to keep the lightest squark at a mass of $2 \mathrm{TeV}$ for a given $m_{D}^{2}$. Please note that while the limit on the squark mass is reduced for $m_{D}^{2} \gg 0$, cf. section 3.1 , we will use $m_{\tilde{q}}=2 \mathrm{TeV}$ in all cases for easy comparison. The remaining model parameters are thus fixed as

$$
\begin{aligned}
& m_{10_{H}}^{2}=-(3647 \mathrm{GeV})^{2}, \quad m_{1 / 2}=389 \mathrm{GeV}, \\
& A_{0}=-3140 \mathrm{GeV}, \quad \tan \beta=39, \quad \operatorname{sign}\left(\mu_{H}\right)=1, \\
& m_{16_{F}}^{2} \text { such that } \min \left(m_{\tilde{q}}\right)=2 \mathrm{TeV},
\end{aligned}
$$


unless otherwise noted. Figure 5 shows the dependence of the masses on $m_{D}^{2}$ for both scenarios, using the 2-loop RGEs described in Appendix A in Supplementary Material. Most obviously, the splitting between the sparticles in different representations of SU(5) increases with larger values of $\left|m_{D}^{2}\right|$. However, the splitting between the first generation squarks and sleptons does not get big enough for the sleptons to become appreciably lighter before the third generation stops and sbottoms become tachyonic. For both $m_{D}^{2}>0$ and $m_{D}^{2}<0$, the lightest sparticle is the lightest sbottom. The regions with $m_{D}^{2} \gtrsim(1.1 \mathrm{TeV})^{2}$ and $m_{D}^{2} \lesssim-(1.8 \mathrm{TeV})^{2}$ are non-physical. For the case of negative $m_{D}^{2}$ we have obtained, in a rather natural way, very light stops, sbottoms and staus, while the rest of the scalars are above $1 \mathrm{TeV}$. This is consistent with current experimental data $[32,34]$ and would provide a natural solution to the hierarchy problem, with a reasonable fine tuning due to light stops and sbottoms. We have, however, chosen a mass for the gluino fixed at $1 \mathrm{TeV}$ resulting in relatively light neutralinos, $m_{\tilde{\chi}_{1}^{0}} \approx 150 \mathrm{GeV}$. In addition to the low energy sparticle masses, Figure 5 also shows the derived value of the Higgs $\mu_{H}$ term, and the soft mass $m_{16_{F}}$ at the GUT scale, respectively. An example sparticle spectrum for this scenario is shown in Figure 10 (left) for $m_{D}^{2}=-(1.83 \mathrm{TeV})^{2}$.

The impact of different values for $m_{1 / 2}$ can be seen in Figure 6 (left) where the allowed $\left(m_{D}^{2}, m_{1 / 2}\right)$ space is shown. Also displayed are the lightest slepton $m_{\tilde{e}}$, the lightest stau $m_{\tilde{\tau}_{1}}$ and the lightest sbottom $m_{\tilde{b}_{1}}$ mass. The outer, shaded (brown) area is excluded because there is at least one tachyonic state, usually the sbottom. The enclosing (orange) band denotes the parameter space where the neutralino $\tilde{\chi}_{1}^{0}$ is not the LSP. The bottom (blue) band is excluded by the gluino mass limit from the direct searches described in section $3,\left(m_{\tilde{g}} \gtrsim 1.1 \mathrm{TeV}\right)$. We can clearly see that increasing $m_{1 / 2}$ has the effect of lowering the masses of all the affected sparticles, particularly the sleptons, cf. Equation (18). However, the mass of the lightest neutralino increases with $m_{1 / 2}$, and for $m_{\tilde{x}_{1}^{0}} \approx 0.4 \mathrm{TeV}$, one of $\tilde{\tau}_{1}, \tilde{e}$, or $\tilde{b}_{1}$ becomes lighter. For $m_{1 / 2}$ close to the upper limit, $m_{1 / 2} \approx 0.9 \mathrm{TeV}$, either the lightest stau or selectron is the NLSP.

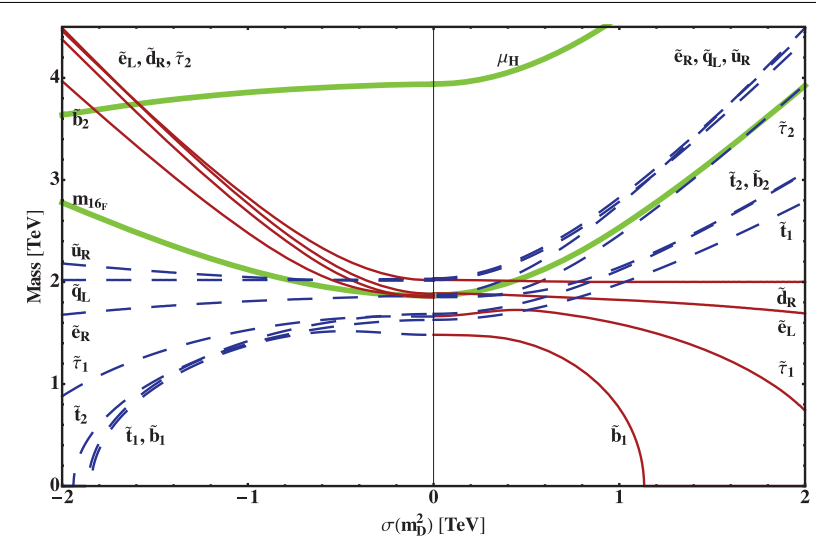

FIGURE 5 | Sparticle masses as a function of $\sigma\left(m_{D}^{2}\right)=\operatorname{sign}\left(m_{D}^{2}\right)$ $\sqrt{\left|\boldsymbol{m}_{D}^{2}\right|}$. The remaining model parameters are fixed as described in Equation (22).
In order to have a better understanding why the third generation squarks are so light compared to their first and second generation counterparts, Figure 6 (right) displays the corresponding properties in the $\left(m_{D}^{2}, A_{0}\right)$ parameter plane. Notice that for the sbottom and the stau, the effects of large $m_{D}^{2}$ and large $A_{0}$ are similar, i.e., they both push the masses down. As a matter of fact, we can actually see that the sbottom is only the lightest for large $A_{0}$ (as was the case in Figure 5), but is heavier than the stau for small $A_{0}$, and can even be rather heavy $\left(m_{\tilde{b}_{1}} \approx 2.4 \mathrm{TeV}\right)$. The effect of $A_{0}$ on the first and second generation slepton mass is negligible due to the small Yukawa couplings, and we do not show it in the plot.

\subsection{LIGHT FIRST GENERATION}

As described above, the lightest sbottom and stop generically constitute the lightest sfermion states, except for large values of $m_{1 / 2}$ and $\left|m_{D}^{2}\right|$. The well known reason for this suppression, also with respect to the first two squark generations, are the large third generation Yukawa couplings which drive the masses down through RGE running. If we look into the terms in the RGEs proportional to the Yukawa couplings (see Appendix A in Supplementary Material), we find that they have the following dependence at the one loop level,

$$
\Delta_{\tau, b, t} \propto m_{10_{H}}^{2}+2 m_{16_{F}}^{2}+A_{0}^{2} .
$$

Hence, in order to minimize this contribution, we need to compensate the increasingly large values of $m_{16_{F}}^{2}$ with equally large and opposite sign values of $m_{10_{H}}^{2}+A_{0}^{2}$. If we want to keep the trilinear couplings real, the best choice for this would be $A_{0}=0$ and $m_{10_{H}}^{2}=-2 m_{16_{F}}^{2}$. Including two loop corrections to the masses, one needs to increase this proportionality by about $5-10 \%$ to compensate the suppression of the stau, stop and sbottoms masses with respect to the first two generations. In the following we will use the relation $m_{10_{H}}^{2}=-2.1 m_{16_{F}}^{2}$. This clearly defines a rather fine-tuned solution as the Yukawa couplings are a priori unrelated to the soft SUSY breaking parameter. We nevertheless study this case as an extreme departure from the generic picture described in section 4.1. In summary, the base model parameters used in this section are described by

$$
\begin{aligned}
m_{1 / 2} & =389 \mathrm{GeV}, \\
A_{0} & =0 \quad \tan \beta=39, \quad \operatorname{sign}\left(\mu_{H}\right)=1, \\
m_{16_{F}}^{2}, m_{10_{H}}^{2} & =-2.1 m_{16_{F}}^{2} \text { such that } \min \left(m_{\tilde{q}}\right)=2 \mathrm{TeV},
\end{aligned}
$$

unless otherwise noted. Figure 7 shows the effect of approximately compensating the third generation Yukawa couplings on the sparticle masses. We see that indeed the third generation sparticles are heavier than their first generation counterparts. In comparison with Figure 5, the $\mathrm{SO}(10) \mathrm{D}$-term $m_{D}^{2}$ can be larger, up to $m_{D}^{2} \lesssim(5 \mathrm{TeV})^{2}$, in turn producing a wider splitting between the lightest squarks and the lightest sleptons. On the other hand, the heavy squarks and sleptons would be split off considerably, with masses up to $10 \mathrm{TeV}$. This is a clear example of a SplitSUSY [45-48] scenario, exhibiting a three-fold splitting: Very light sleptons $\approx 0.1-0.2 \mathrm{TeV}$, lightest squarks around $2-4 \mathrm{TeV}$ and 


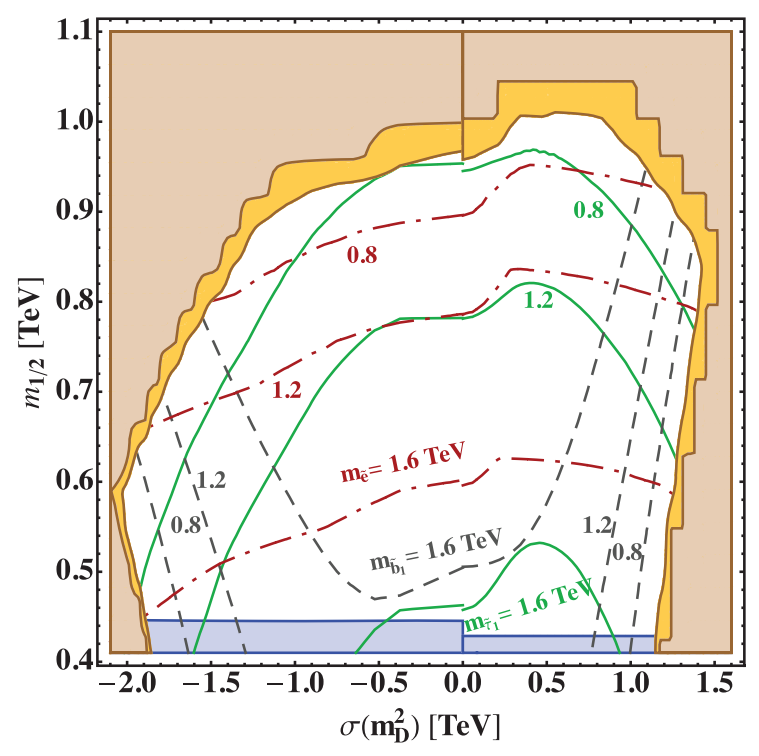

FIGURE 6 | Mass of the lightest stau $\tilde{\boldsymbol{\tau}}_{1}$ (solid green), sbottom $\tilde{b}_{1}$ (dashed gray), and selectron $\tilde{e}$ (dash-dotted red) as a function of $m_{D}^{2}$ and $m_{1 / 2}$ (left) and of $m_{D}^{2}$ and $A_{0}$ (right). The remaining parameters are respectively fixed as described in

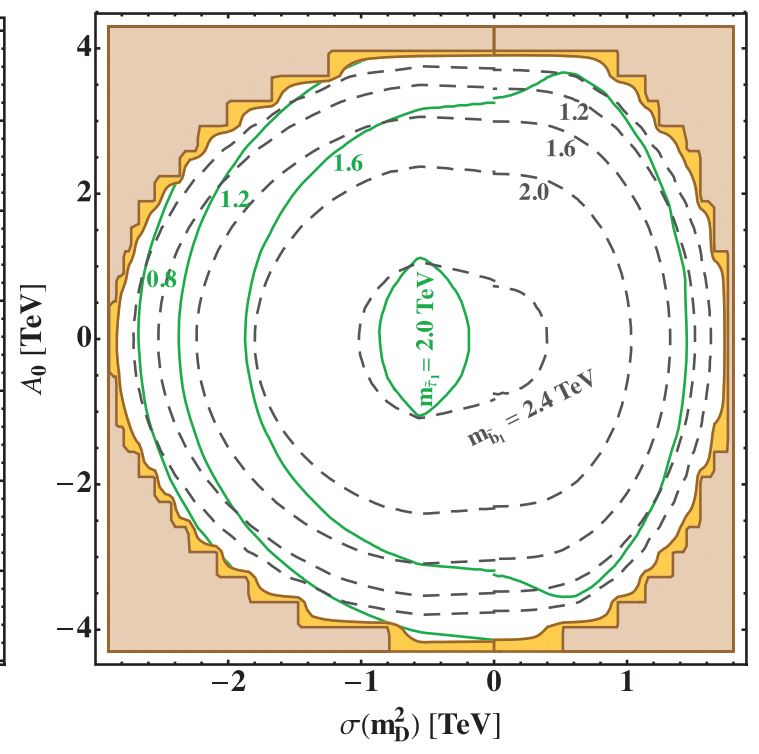

Equation (22). The colored areas are excluded or disfavored because there is at least one tachyonic state (brown), the neutralino is not the LSP (orange), the gluino mass is below the experimental limit (blue).

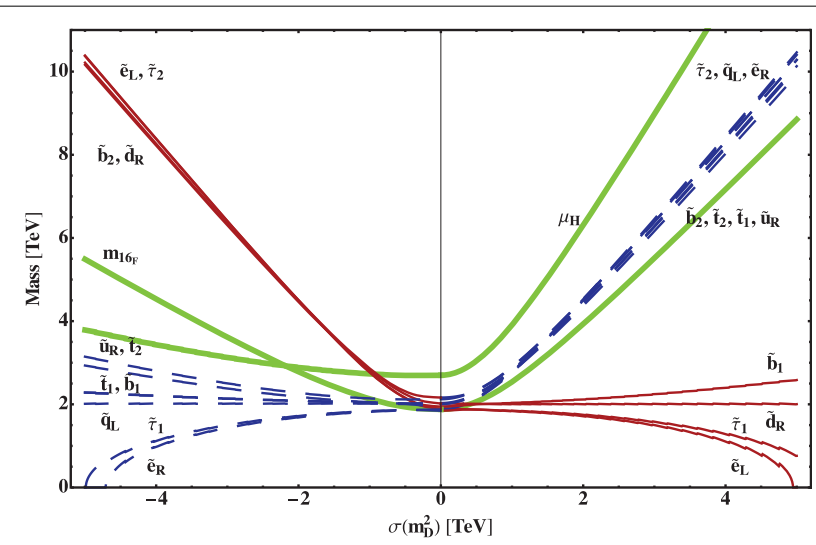

FIGURE 7 | As Figure 5, but with the remaining model parameters fixed as described in Equation (24).

very heavy squarks and sleptons at 9-10 TeV. An example sparticle spectrum for this scenario is shown in Figure 10 (right) for $m_{D}^{2}=+(4.87 \mathrm{TeV})^{2}$.

The combined dependencies on $m_{D}^{2}$ and either $m_{1 / 2}$ or $A_{0}$ is displayed in Figure 8. The excluded or disfavored shaded areas are defined as before in Figure 6. We do not plot the lightest sbottom mass in Figure 8 (left) as it is too heavy to be of interest here. The main difference from the light third generation case displayed in Figure 8 is that the first generation sleptons are slightly lighter than the light stau, except for small values of $\left|m_{D}^{2}\right|$. Due to the potentially higher values of $\left|m_{D}^{2}\right|$, very small slepton masses are possible even for low values of $m_{1 / 2}$.
The dependence on $A_{0}$, Figure 8 (right) in this case is also rather different from Figure 6 (right). While the stau mass exhibits a similar behavior, the sbottom mass becomes heavier with increasing $\left|m_{D}^{2}\right|$ but lighter with increasing $A_{0}$. This is expected as we do not compensate the effect of $A_{0}$ on the Yukawadriven RGE contributions. As a consequence, the lightest sbottom will become the lightest sfermion for large $A_{0} \gtrsim 3 \mathrm{TeV}$.

The scenario described here would be optimal for sleptons searches at LHC because it allows for very light first, second and also third generation sleptons. Naively, one might expect that the presence of very light (left-handed) smuons is able to enhance the predicted value of the anomalous magnetic moment of the muon closer to the experimentally favored value, $\Delta a_{\mu} \equiv a_{\mu}^{\exp }-a_{\mu}^{\mathrm{SM}}=$ $(26.1 \pm 8.0) \times 10^{-10}$ [49]. This is because the supersymmetric contributions to $a_{\mu}$ are driven by muon sneutrino-chargino and smuon-neutralino loops. Unfortunately, the SUSY scenarios considered here require a large Higgs $\mu$-term $\mu_{H}$ as shown in Figures 5, 7. For a strongly split scenario as in our case, the SUSY contribution is roughly $[50,51]$

$$
\Delta a_{\mu}^{\mathrm{SUSY}} \lesssim 10^{-8} \times \frac{\tan \beta}{10} \frac{(100 \mathrm{GeV})^{2}}{M_{1} \mu_{H}},
$$

with the lightest gaugino mass $M_{1}$. Consequently, a strongly split scenario with large $\left|m_{D}^{2}\right|$ in minimal SUSY SO(10) does not enhance $\Delta a_{\mu}^{\text {SUSY }}$ appreciably compared to the standard CMSSM case.

\subsection{NON-UNIVERSAL GAUGINOS}

As a final step of our analysis, we will briefly comment on the impact of non-universal gauginos at the GUT scale. In Table 1 

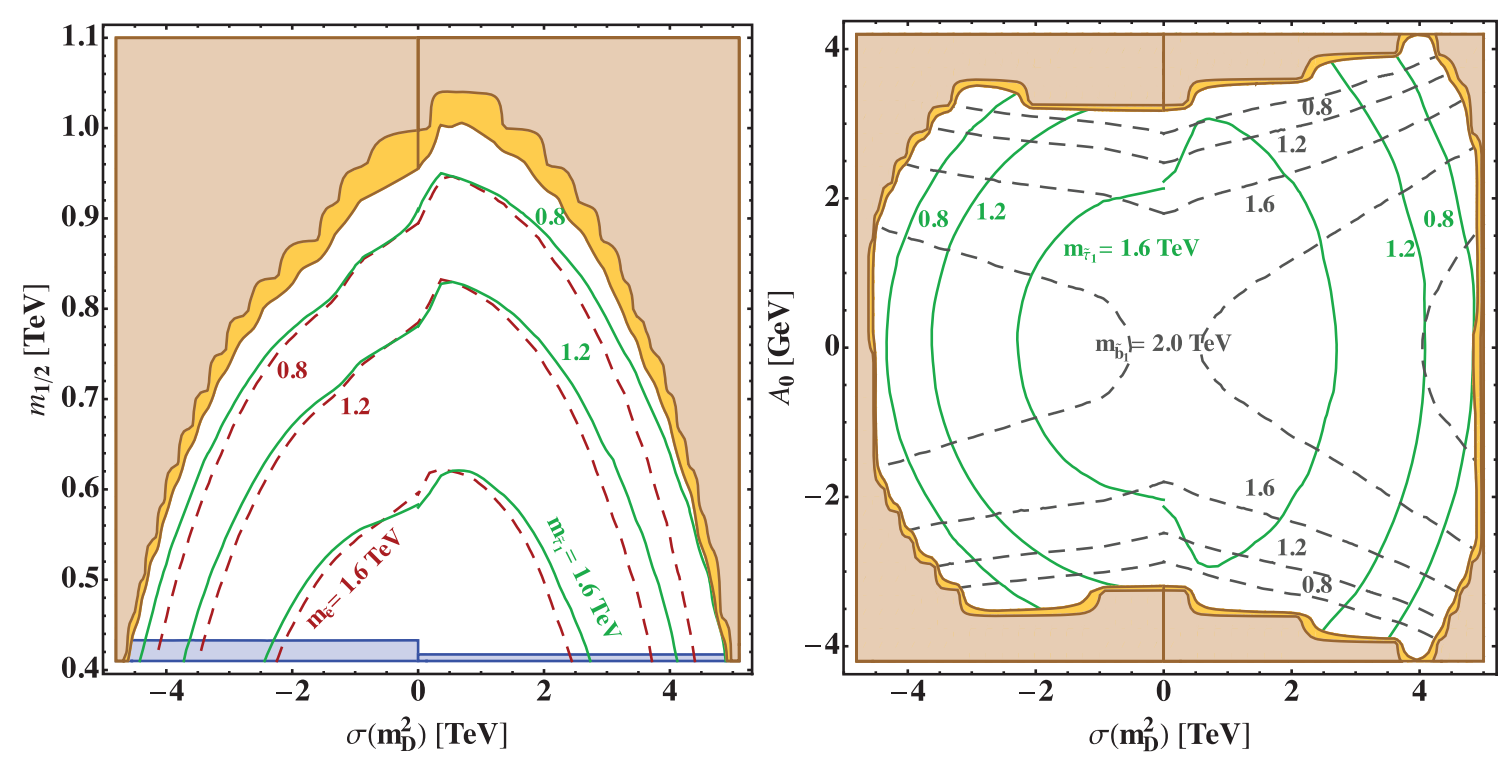

FIGURE 8 | As Figure 6, but with the remaining model parameters fixed as described in Equation (24).

we see that there are three representative cases: (a) The messenger field is in the singlet representation of the SU(5) embedded in $\mathrm{SO}(10)$. This corresponds to the standard universal case with an approximate gaugino hierarchy of $\left|M_{1}\right|:\left|M_{2}\right|:\left|M_{3}\right|=1 / 6$ : $1 / 3: 1$ near the EW scale, which we have discussed above. (b) The messenger is in the 24-dimensional representation. Here, the bino is comparatively lighter than in the CMSSM, with an approximate gaugino hierarchy of $\left|M_{1}\right|:\left|M_{2}\right|:\left|M_{3}\right|=1 / 12: 1 / 2: 1$ near the EW scale. This is phenomenologically interesting as it creates a larger splitting between the lightest neutralino (essentially the bino) and the gluino. It potentially permits a very light neutralino while satisfying the direct gluino mass limits, cf. section 3. For example, for a gluino mass at the current limit, $m_{\tilde{g}} \approx 1.1 \mathrm{TeV}$, the lightest neutralino could be lighter than $m_{\tilde{\chi}_{1}^{0}} \approx$ $100 \mathrm{GeV}$, subject to direct search limits, cf. section 3.2. On the other hand, the ratio between $M_{2}$ and $M_{3}$ is smaller than that of normal CMSSM, making the second neutralino and lightest chargino slightly heavier. Such a change will for instance suppress the SUSY contribution to the anomalous magnetic moment of the muon. The largest contribution comes from a sneutrinochargino loop, and the experimental situation would prefer both the $\mathrm{SU}(2)$ gaugino and the sleptons to be light. (c) The messenger is in the 200-dimensional representation, corresponding to a low energy hierarchy $\left|M_{1}\right|:\left|M_{2}\right|:\left|M_{3}\right|=5 / 3: 2 / 3: 1$. The spectrum is rather different here, with the bino being the heaviest gaugino, while the mass of the wino is approximately $2 / 3$ of the gluino mass. Hence, the lightest neutralino would be mostly wino and would have a relatively large mass for a given gluino mass, compared to the previous case.

Other than the direct effect on the gaugino masses, the presence of non-universal gauginos at the GUT scale will also affect the masses of the scalar SUSY particles due to the impact on the RGE running. So far we have calculated scalar particle masses assuming degenerate gauginos at the GUT scale, resulting in a term $\propto m_{1 / 2}^{2}$ as the main RGE effect on the scalar masses, see for example Equation (18). Allowing for arbitrary individual gaugino masses $M_{1}, M_{2}$, and $M_{3}$ at the GUT scale, these equations will take the form

$$
\begin{aligned}
& m_{\tilde{d}_{R}}^{2}-m_{\tilde{e}_{L}}^{2}=0.2 m_{D}^{2}-0.02 M_{1}^{2}-0.5 M_{2}^{2}+4.9 M_{3}^{2}+\mathcal{O}\left(M_{Z}^{2}\right), \\
& m_{\tilde{u}_{L}}^{2}-m_{\tilde{e}_{R}}^{2}=-0.2 m_{D}^{2}-0.15 M_{1}^{2}+0.5 M_{2}^{2}+4.9 M_{3}^{2}+\mathcal{O}\left(M_{Z}^{2}\right), \\
& m_{\tilde{u}_{R}}^{2}-m_{\tilde{e}_{R}}^{2}=-0.3 m_{D}^{2}-0.08 M_{1}^{2}+4.8 M_{3}^{2}+\mathcal{O}\left(M_{Z}^{2}\right)
\end{aligned}
$$

By far the largest contribution is due to the strong gauge effect of the gluino affecting the squarks. In fixing the gluino mass as $m_{\tilde{g}} \approx 1.1 \mathrm{TeV}$ in tune with the experimental bound, we essentially set the scale of the absolute squark masses. The gaugino non-universality will then induce an additional splitting between the squarks and sleptons, dominantly driven by the wino mass $M_{2}$. A comparison of the three cases is shown in Figure 9, i.e., (a) universal gauginos (solid), (b) light bino case (short dashed) and (c) light wino case (long dashed). As expected from Equation (26), case (b) produces only small deviations when compared to universal gauginos. On the other hand, case (c) can have a sizable impact on the slepton masses, especially for $m_{D}^{2}<0$. The negative signs in front of $M_{1}^{2}$ in Equation (26) explain the larger slepton masses compared to the universal gaugino case.

\section{CONCLUSIONS}

Supersymmetric models are feeling the pinch from the lack of new physics signals at the LHC and in low energy observables. While any phenomenological limits can be evaded by sending the SUSY particle masses to higher scales, such a solution will usually negate the ability of many SUSY models to solve the hierarchy problem of the Standard Model. Minimal scenarios, such as the CMSSM are especially difficult in this regard as the stringent lower limits from LHC direct searches on colored states will 
similarly affect non-colored sparticles. As a consequence, there is now much effort going into the study of less constrained models of low energy SUSY with a large variety of spectra. For example, phenomenological approaches like the phenomenological MSSM do not contain a priori relations between different sparticle species.

In this work, we focused on the other hand on a minimal supersymmetric $\mathrm{SO}(10)$ model incorporating one-step symmetry breaking from $\mathrm{SO}(10)$ down to the Standard Model gauge group at the usual GUT scale. Such SUSY GUT scenarios are of course very well motivated with the possibility of unifying the gauge and Yukawa couplings at the GUT scale. With respect to the SUSY spectrum, the GUT unification also provides a motivation

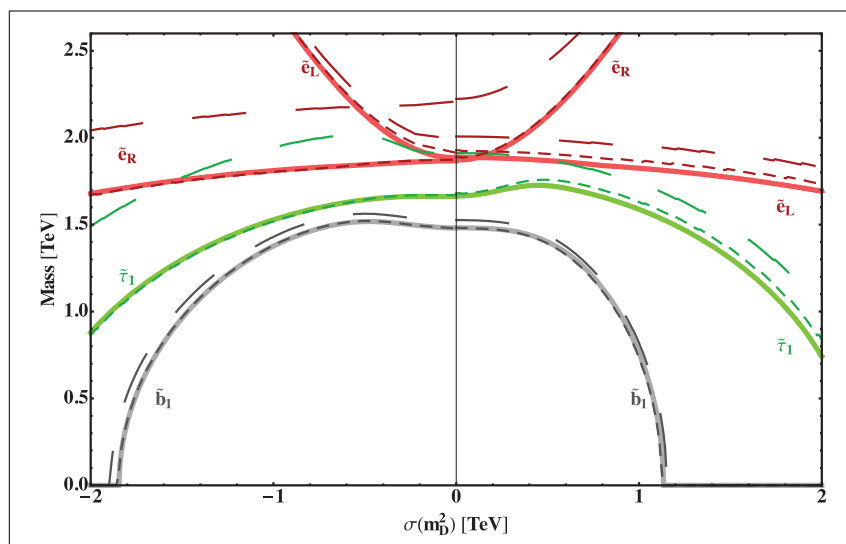

FIGURE 9 | Sparticle masses as a function of $\sigma\left(m_{D}^{2}\right)=\operatorname{sign}\left(m_{D}^{2}\right) \sqrt{\left|m_{D}^{2}\right|}$. The remaining model parameters are fixed as described in Equation (22) for three different gaugino hierarchies at the GUT scale: (a) $M_{1}=M_{2}=M_{3}=$ $m_{1 / 2}$ (universality, solid); (b) $-2 M_{1}=-3 / 2 M_{2}=M_{3}=m_{1 / 2}$ (light bino, short dashed); (c) $10 M_{1}=2 M_{2}=M_{3}=m_{1 / 2}$ (light wino, long dashed). for the degeneracy of the soft SUSY breaking masses and couplings. In contrast to the CMSSM though, the scalar masses in an $\mathrm{SO}(10)$ GUT are shifted by D-terms associated with the breaking of $\mathrm{SO}(10)$ to the lower-rank SM group. These D-terms do depend on the details of the gauge breaking but are generally expected to be of the order of the SUSY breaking scale (for example described by SUSY breaking mass $m_{16_{F}}^{2}$ of the matter $\mathrm{SO}(10)$ 16-plet), and can be parametrized by a single additional quantity $m_{D}^{2}$. This provides a controlled departure from the degeneracy of the CMSSM. In addition, we also briefly discuss the possibility of non-universal gaugino masses at the GUT. This is a general possibility in SUSY GUT models with gravity mediated breaking if the SUSY breaking messenger is not a singlet under the GUT gauge group.

We have considered three scenarios: Firstly, starting from a non-universal Higgs mass benchmark scenario, cf. Equation (22), we studied the impact of the D-term $m_{D}^{2}$ on the sparticle spectrum, especially on the possibility to obtain light third generation squarks and sleptons. In particular, we found that for $m_{D}^{2} \lesssim-m_{16_{F}}^{2}$, both stops, the lightest sbottom and the lightest stau can be very light, while the first generation squarks and sleptons are heavy. An example spectrum is shown in Figure 10 (left) for $m_{D}^{2} \approx-(1.8 \mathrm{TeV})^{2} \approx-0.5 \times m_{16_{F}}^{2}$. Such a spectrum can be viable as a solution to the hierarchy problem as it keeps the fine tuning under control. It belongs to a class of Split-SUSY scenarios with a compressed spectrum [52-54], with the lightest stop too light to decay into a top and the lightest neutralino. The LHC limit on the stop mass for this case is much more relaxed that in other scenarios. With a light stop mass just above the LHC limit for a compressed spectrum, $m_{\tilde{t}_{1}} \gtrsim 250 \mathrm{GeV}$, a rough estimate of the fine tuning would be $M_{\mathrm{SUSY}}^{2} / m_{t}^{2} \approx m_{\tilde{t}_{1}} m_{\tilde{t}_{2}} / m_{t}^{2} \approx 5$.

Secondly, we extended the previous case to make the first generation light, by way of changing the soft Higgs mass $m_{10_{H}}^{2}$. While this presents a rather extreme scenario which is fine-tuned to cancel the Yukawa contribution of the third generation states,
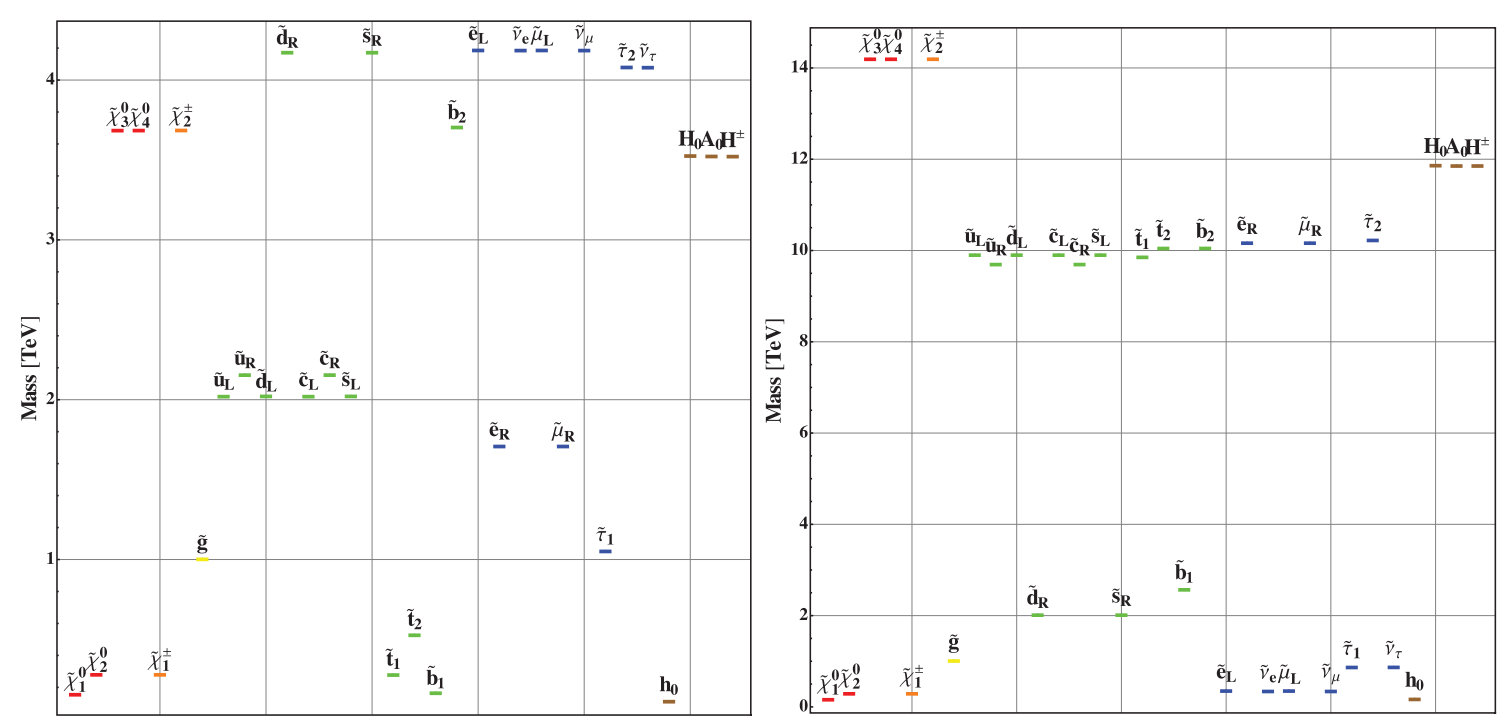

FIGURE 10 | Supersymmetric particle spectra in two example scenarios with large SO(10) D-terms based on Equation (22) with $m_{D}^{2}=-(1.83 \mathrm{TeV})^{2}$ (light third generation, left) and based on Equation (24) with $m_{D}^{2}=+(4.87 \mathrm{TeV})^{2}$ (light first generation, right). 
it demonstrates the potential to deviate from the usual light stop/sbottom/stau case (although this is usually preferred due to naturalness considerations). The direct LHC limits on first and second generation slepton masses are still comparatively weak and can accommodate light sleptons $m_{\tilde{l}} \gtrsim 300 \mathrm{GeV}$. An example spectrum for this case is shown in Figure 10 (right) for $m_{D}^{2} \approx+(4.9 \mathrm{TeV})^{2} \approx 0.3 \times m_{16_{F}}^{2}$, resulting in a severely split scenario. Consequently, it requires a considerable fine-tuning, not only by manually engineering the light selectrons, but also due to the necessary cancelations of the large contributions to the Higgs mass from the heavy stops, $M_{\text {SUSY }}^{2} / m_{t}^{2} \approx m_{\tilde{t}_{1}} m_{\tilde{t}_{2}} / m_{t}^{2} \approx$ $3 \times 10^{3}$. As mentioned, the main purpose of the two limiting examples provided here is to define a rough range of possible spectra in the minimal SUSY SO(10) model with large D-terms. If taken seriously, a spectrum with light first generation sleptons would naively be advantageous to explain the apparent discrepancy between the measured value of the anomalous magnetic moment of the muon $a_{\mu}$ and its SM prediction. Unfortunately, due to the splitting between left- and right-handed smuons in combination with the large Higgs $\mu$-term, it is not possible to appreciably raise the SUSY contribution to $a_{\mu}$. For $m_{D}^{2} \gg 0$, only the right-handed down-type squarks will be light and, as we have demonstrated, this weakens the current direct LHC limit on the corresponding squark masses from $m_{\tilde{q}} \gtrsim 2 \mathrm{TeV}$ to $m_{\tilde{q}} \gtrsim 1 \mathrm{TeV}$.

Finally, we have also briefly looked at the case of non-universal gauginos at the GUT scale. In addition to the universal case, we studied two different choices for the representation of the messenger fields; one where the messenger is in the 24 representation of the SU(5) subgroup embedded in $\mathrm{SO}(10)$, and one where it is in the $\mathbf{2 0 0}$ representation. The former leads to a lighter, binolike lightest neutralino, but it negligibly affects the scalar particle masses. The latter case, leading to bino heavier than the gluino and a wino-like lightest neutralino, has a greater impact on the scalar SUSY particle masses. Both cases can of course affect the possible decay channels and therefore the visible signatures in detail. For example, raising the neutralino masses will facilitate the realization of compressed spectra and the possibility of stopneutralino co-annihilation affecting the dark matter relic density of the universe.

\section{ACKNOWLEDGMENTS}

The work was supported partly by the London Centre for Terauniverse Studies (LCTS), using funding from the European Research Council via the Advanced Investigator Grant 267352. Nishita Desai acknowledges support from the Alexander von Humboldt foundation. Frank F. Deppisch and Tomás E. Gonzalo would like to thank Ben Allanach, Julia Harz, and Werner Porod, Nishita Desai would like to thank Jad Marrouche for useful discussions.

\section{SUPPLEMENTARY MATERIAL}

The Supplementary Material for this article can be found online at: http://www.frontiersin.org/journal/10.3389/fphy.2014.00027/ abstract

\section{REFERENCES}

1. Fritzsch H, Minkowski P. Unified interactions of leptons and hadrons. Annals Phys. (1975) 93:193-266. doi: 10.1016/0003-4916(75)90211-0
2. Anderson G, Raby S, Dimopoulos S, Hall LJ, Starkman GD. A systematic SO(10) operator analysis for fermion masses. Phys Rev. (1994) D49:3660-90.

3. Raby S. Phenomenology of the minimal SO(10) SUSY model. Pramana. (2004) 62:523-36. doi: 10.1007/BF02705106

4. Aulakh CS. Pinning down the new minimal supersymmetric GUT. Phys Lett. (2008) B661:196-200. doi: 10.1016/j.physletb.2008.01.053

5. Grimus W, Kuhbock H. A renormalizable SO(10) GUT scenario with spontaneous CP violation. Eur Phys J. (2007) C51:721-9. doi: 10.1140/epjc/s10052007-0324-5

6. Aulakh CS, Garg SK. The new minimal supersymmetric GUT: spectra, RG analysis and fermion fits. Nucl Phys B. (2006) 857:101-142.

7. Aulakh CS. MSGUT reborn? (2006). arXiv:hep-ph/0607252

8. Wiesenfeldt S, Willenbrock S. Perturbative SO(10) GUT and the minimal Higgs sector. Phys Lett. (2008) B661:268-72. doi: 10.1016/j.physletb.2008. 02.022

9. Nath P, Fileviez Perez P. Proton stability in grand unified theories, in strings and in branes. Phys Rept. (2007) 441:191-317. doi: 10.1016/j.physrep.2007.02.010

10. Drees M. Intermediate scale symmetry breaking and the spectrum of super partners in superstring inspired supergravity models. Phys Lett. (1986) B181:279. doi: 10.1016/0370-2693(86)90046-8

11. Kolda CF, Martin SP. Low-energy supersymmetry with D term contributions to scalar masses. Phys Rev. (1996) D53:3871-83.

12. Baer H, Diaz MA, Quintana P, Tata X. Impact of physical principles at very high-energy scales on the superparticle mass spectrum. JHEP. (2000) 0004:016.

13. Jack I, Jones DRT, Kord AF. Three loop soft running, benchmark points and semiperturbative unification. Phys Lett. (2004) B579:180-8. doi: 10.1016/j.physletb.2003.10.083

14. Aguilar-Saavedra JA, Ali A, Allanach BC, Arnowitt RL, Baer HA, Bagger JA. et al. Supersymmetry parameter analysis: SPA convention and project. Eur Phys J. (2006) C46:43-60. doi: 10.1140/epjc/s2005-02460-1

15. Freitas A, Porod W, Zerwas PM. Determining sneutrino masses and physical implications. Phys Rev. (2005) D72:115002. doi: 10.1103/PhysRevD.72.115002

16. Blair GA, Porod W, Zerwas PM. Reconstructing supersymmetric theories at high-energy scales. Phys Rev. (2001) D63:017703.

17. Blair GA, Porod W, Zerwas PM. The reconstruction of supersymmetric theories at high-energy scales. Eur Phys J. (2003) C27:263-81. doi: 10.1103/PhysRevD.63.017703

18. Deppisch F, Freitas A, Porod W, Zerwas PM. Determining heavy mass parameters in supersymmetric SO(10) models. Phys Rev. (2008) D77:075009.

19. Deppisch F. Determining heavy mass parameters in SUSY SO(10). eConf. (2007) C0705302:SUS02.

20. Miller DJ, Morais AP, Pandita PN. Constraining grand unification using first and second generation sfermions. Phys Rev. (2013) D87:015007. doi: 10.1103/PhysRevD.87.015007

21. Miller DJ, Morais AP. Supersymmetric SU(5) grand Unification for a post Higgs Boson Era. JHEP. (2013) 1310:226. doi: 10.1007/JHEP10(2013)226

22. Martin SP. Non-universal gaugino masses from non-singlet F-terms in non-minimal unified models. Phys Rev. (2009) 79:095019. doi: 10.1103/PhysRevD.79.095019

23. Drees M, Godbole R, Roy P. Theory and Phenomenology of Sparticles. Singapore: World Scientific (2004).

24. Buchmueller O, Cavanaugh R, De Roeck A, Dolan MJ, Ellis JR, Flacher H, et al. The CMSSM and NUHM1 after LHC Run 1. (2013).

25. Badziak M. Yukawa unification in SUSY SO(10) in light of the LHC Higgs data. Mod Phys Lett A. (2012) 27:1230020. doi: 10.1142/S0217732312300200

26. Dine M, Nelson AE. Dynamical supersymmetry breaking at low-energies. Phys Rev. (1993) D48:1277-87.

27. The ATLAS collaboration. Search for squarks and gluinos with the ATLAS detector in final states with jets and missing transverse momentum and 20.3 $\mathrm{fb}^{-1}$ of $\sqrt{s}=8 \mathrm{TeV}$ proton-proton collision data. Geneva: CERN (2013). ATLAS-CONF-2013-047.

28. Chatrchyan S, Khachatryan V, Sirunyan AM, Tumasyan A, Adam W, Bergauer, T. Search for new physics in the multijet and missing transverse momentum final state in proton-proton collisions at $\operatorname{sqrt}(\mathrm{s})=8 \mathrm{TeV}$ (2014). arXiv:1402.4770. CMS-SUS-13-012, CERN-PH-EP-2014-015

29. Sjostrand T, Mrenna S, Skands PZ. PYTHIA 6.4 physics and manual. JHEP. (2006) 0605:026. doi: 10.1088/1126-6708/2006/05/026 
30. Sjostrand T, Mrenna S, Skands PZ. A brief introduction to PYTHIA 8.1. Comput Phys Commun. (2008) 178:852-67. doi: 10.1016/j.cpc.2008. 01.036

31. Desai N, Skands PZ. Supersymmetry and generic BSM models in PYTHIA 8. Eur Phys J. (2012) C72:2238. doi: 10.1140/epjc/s10052-012-2238-0

32. ATLAS Collaboration. Geneva: CERN (2013). ATLAS-CONF-2013-024, ATLAS-CONF-2013-037, ATLAS-CONF-2013-048, ATLAS-CONF-2013-065, ATLAS-CONF-2013-068.

33. Aad G, Abajyan T, Abbott B, Abdallah J, Khalek SA, Abdinov O. Search for direct third-generation squark pair production in final states with missing transverse momentum and two $b$-jets in $\sqrt{s}=8 \mathrm{TeV} p p$ collisions with the ATLAS detector. JHEP. (2013) 1310:189. doi: 10.1007/JHEP10(2013)189

34. Chatrchyan S, Khachatryan V, Sirunyan AM, Tumasyan A, Adam W, Bergauer T, Search for top-squark pair production in the single-lepton final state in pp collisions at $\sqrt{s}=8$ TeV. Eur Phys J. (2013) C73:2677. doi: 10.1140/epjc/s10052-013-2677-2

35. CMS Collaboration. Search for supersymmetry in pp collisions at $\operatorname{sqrt}(\mathrm{s})=8$ $\mathrm{TeV}$ in events with three leptons and at least one b-tagged jet. Geneva: CERN (2013). CMS-PAS-SUS-13-008.

36. Chatrchyan S, Khachatryan V, Sirunyan AM, Tumasyan A, Adam W, Bergauer T. Search for supersymmetry in hadronic final states with missing transverse energy using the variables AlphaT and b-quark multiplicity in pp collisions at 8 TeV. Eur Phys J. (2013) C73:2568. doi: 10.1140/epjc/s10052-013-2568-6

37. ATLAS Collaboration. Geneva: CERN (2013). ATLAS-CONF-2013028,ATLAS-CONF-2013-035.

38. ATLAS Collaboration. Geneva: CERN (2013). ATLAS-CONF-2013-049.

39. CMS Collaboration. Geneva: CERN (2013). CMS-PAS-SUS-13-006.

40. CMS Collaboration. Geneva: CERN (2013). CMS-PAS-SUS-13-017.

41. Aad G, Abajyan T, Abbott B, Abdallah J, Khalek SA, Abdinov, O. Search for the neutral Higgs bosons of the Minimal Supersymmetric Standard Model in $p p$ collisions at $\sqrt{s}=7 \mathrm{TeV}$ with the ATLAS detector. JHEP. (2013) 1302:095. doi: 10.1007/JHEP02(2013)095

42. ATLAS Collaboration. Search for charged Higgs bosons in the $\tau+$ jets final state with pp collision data recorded at $\sqrt{s}=8 \mathrm{TeV}$ with the ATLAS experiment. Geneva: CERN (2013). ATLAS-CONF-2013-090.

43. Chatrchyan S, Khachatryan V, Sirunyan AM, Tumasyan A, Adam W, Bergauer T. Search for a light charged Higgs boson in top quark decays in $p p$ collisions at $\sqrt{s}=7 \mathrm{TeV}$. JHEP. (2012) 1207:143.
44. Higgs to tau tau (MSSM). Geneva: CERN (2013). CMS-PAS-HIG-13-021.

45. Arkani-Hamed N, Dimopoulos S. Supersymmetric unification without low energy supersymmetry and signatures for fine-tuning at the LHC. JHEP. (2005) 0506:073

46. Giudice GF, Romanino A. Split supersymmetry. Nucl Phys. (2004) B699:65-89.

47. Arkani-Hamed N, Gupta A, Kaplan DE, Weiner N, Zorawski T. Simply Unnatural Supersymmetry (2012). arXiv:1212.6971.

48. Arvanitaki A, Craig N, Dimopoulos S, Villadoro G. Mini-split. JHEP. (2013) 1302:126. doi: 10.1007/JHEP02(2013)126

49. Davier M, Hoecker A, Malaescu B, Zhang Z. Reevaluation of the hadronic contributions to the Muon g-2 and to alpha(MZ). Eur Phys J. (2011) C71:1515.

50. Moroi T. The Muon anomalous magnetic dipole moment in the minimal supersymmetric standard model. Phys Rev. (1996) D53:6565-75.

51. Endo M, Hamaguchi K, Iwamoto S, Yoshinaga T. Muon $g-2$ vs LHC in supersymmetric models. JHEP. (2014) 1401:123. doi: 10.1007/JHEP01 (2014) 123

52. Alwall J, Le MP, Lisanti M, Wacker JG. Searching for directly decaying gluinos at the tevatron. Phys Lett. (2008) B666:34-7. doi: 10.1016/j.physletb.2008. 06.065

53. LeCompte TJ, Martin SP. Large Hadron Collider reach for supersymmetric models with compressed mass spectra. Phys Rev. (2011) D84:015004.

54. Brümmer F, Kraml S, Kulkarni S, Smith C. The flavour of natural SUSY (2014).

Conflict of Interest Statement: The authors declare that the research was conducted in the absence of any commercial or financial relationships that could be construed as a potential conflict of interest.

Received: 11 March 2014; accepted: 15 April 2014; published online: 06 May 2014. Citation: Deppisch FF, Desai N and Gonzalo TE (2014) Compressed and split spectra in minimal SUSY SO(10). Front. Physics 2:27. doi: 10.3389/fphy.2014.00027

This article was submitted to High-Energy and Astroparticle Physics, a section of the journal Frontiers in Physics.

Copyright (c) 2014 Deppisch, Desai and Gonzalo. This is an open-access article distributed under the terms of the Creative Commons Attribution License (CC BY). The use, distribution or reproduction in other forums is permitted, provided the original author(s) or licensor are credited and that the original publication in this journal is cited, in accordance with accepted academic practice. No use, distribution or reproduction is permitted which does not comply with these terms. 